\title{
Design of a Single Negative Metamaterial based Microstrip Patch Antenna
}

\author{
Amit Gangwar \\ PG student, M.tech Wireless \& mobile \\ communication, DIT, Dehradun, UK India
}

\author{
Suresh Chandra Gupta \\ Professor Department of Electronics \& comm. \\ Engineering, DIT, Dehradun, UK India
}

\begin{abstract}
This paper describes the design and simulation of the new $\mathcal{E}$ negative metamaterial unit cell structure and then the unit cells are combined to form 3D array which use as metamaterial cover with a single microstrip patch antenna. The new $\varepsilon$ negative metamaterial is based on the ELC resonator structure, which is used for obtaining the negative value of permittivity and positive value of permeability. Simulation result shows that the gain of the antenna has been increased with metamaterial cover up to $1.46 \mathrm{db}$. This has to proven that metamaterial as a cover enhance the directive properties of conventional microstrip patch antenna.
\end{abstract}

\section{Keywords}

Metamaterial (MTM), Epsilon negative (ENG) metamaterial, Microstrip Antenna (MSA), Electric-LC (ELC) Resonator, HFSS.

\section{INTRODUCTION}

Metamaterial with unusual electromagnetic properties have attracted a great deal of attraction and attention of researchers and scientists in recent years. Metamaterial are artificially constructed materials which have properties that are not available in naturally occurring materials [1-2]. In the designing of metamaterial most of material properties have to be realized together and these properties are defined by the constitutive parameters permittivity $(\varepsilon)$ and permeability $(\mu)$ .this paper has focused on $\varepsilon$ Negative (ENG) metamaterial which have negative permittivity and positive permeability.

Many researchers have been used this artificial materials for improving the properties of the microwave devices such as antenna and filter. In 2005 a patch antenna with metamaterial cover was proposed and their numerical result showed significant improvement in directivity, compared to conventional patch antenna. Many papers have been published on metamaterial integrated antennas and their analyzed results shown that the metamaterial made a low gain antenna becomes directive with an increment of gain [3-4].

This paper discusses and analyzes the properties of $\varepsilon$ negative metamaterial and the microstrip antenna with and without the $\varepsilon$ negative metamaterial cover. The designed metamaterial structure is a modified ELC resonator structure. It described here in terms of its equivalent circuit also. It is combination of two parallel capacitor connected in parallel with single loop. Where capacitor couples to the electric field and single loop provides inductance to the circuit. The MTM structure and the microstrip antenna are designed to operate at $14.3 \mathrm{GHz}$. The negative permittivity and positive permeability of simulated MTM structure has presented.

\section{PROPOSED STRUCTURE}

\subsection{Design consideration of metamaterial structure}

Figure 1 illustrates the $\varepsilon$ negative metamaterial structure and its dimensions. The ELC resonator structure is printed on a dielectric substrate of thickness $0.203 \mathrm{~mm}$ and dielectric constant 4.4 (FR4).

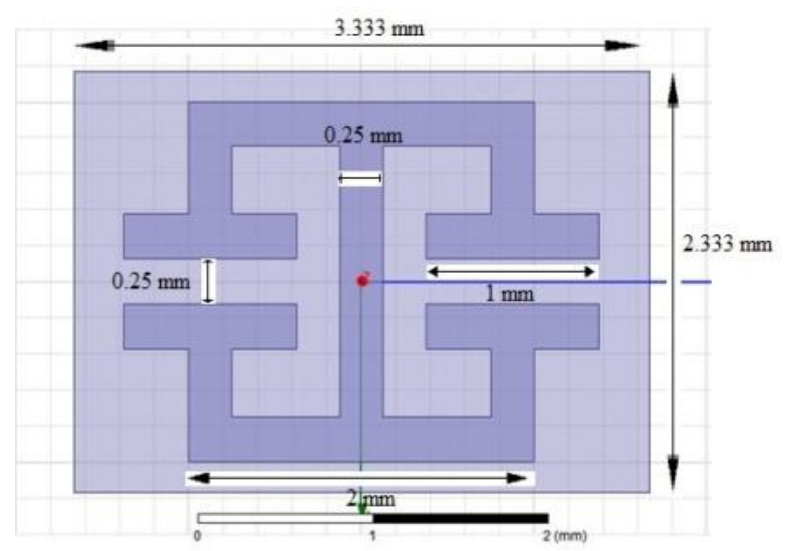

Fig 1: Front view of unit cell

The unit cell is simulated by HFSS by using PEC and PMC boundary condition. The electric field is normal to the "capacitor plates" and thus couples to the capacitive element. The PEC boundary condition is applied to those surfaces which are perpendicular to incident electric field vector.

The structure under investigation is placed in a waveguide with dimension $3.333 \mathrm{~mm} \times 3.333 \mathrm{~mm} \times 11.52 \mathrm{~mm}$ as shown in Figure 2. The results are measured over the frequency range of $8 \mathrm{GHZ}$ to $20 \mathrm{GHZ}$ by EM solver Ansoft HFSS.

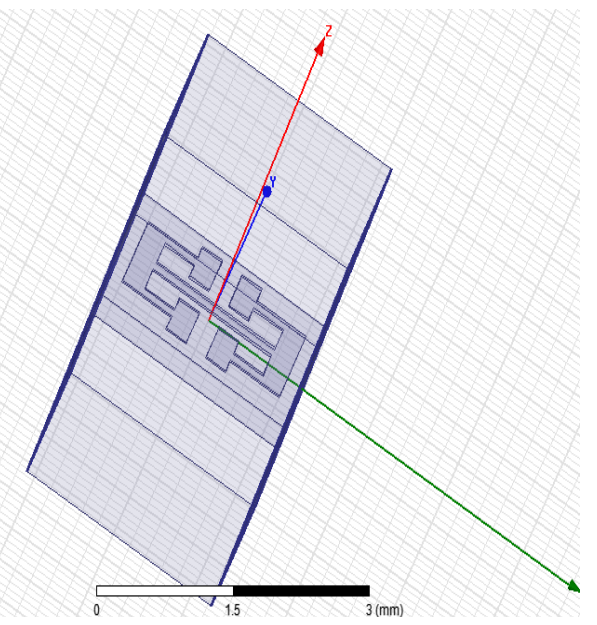

Fig 2: HFSS simulation setup for unit cell structure 


\subsection{Equivalent circuit model}

The ELC resonator can be described qualitatively in terms of its equivalent circuit. Sufficiently accurate equivalent circuit determines the behavior of the structure in a simple, fast and efficient way shown in Figure 3. The ELC resonator has both inductive and capacitive elements like SRR resonator but only the capacitive element couples strongly to the electric field [5]. The resonant frequency of the circuit model can be expressed as $\omega_{0}=\frac{1}{\sqrt{L C}}$ where the equivalent capacitance $C$ and inductance $L$ can be derived using constitutive equations and analytical expressions to calculate the resonant frequency from the various geometrical parameters of the ELC [6-7].

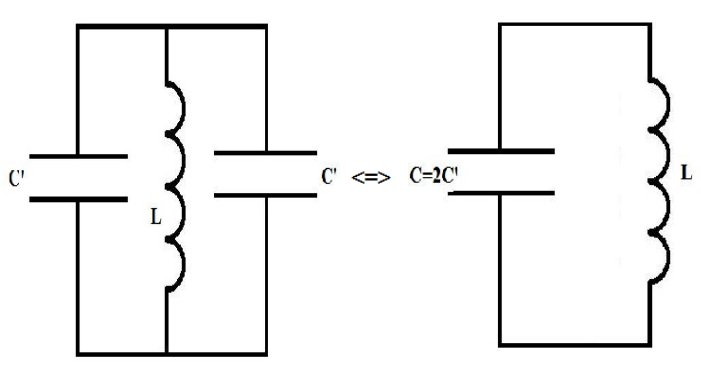

Fig 3: Equivalent circuit representation of ELC

$L=\frac{\mu_{0} l}{2 \pi}\left[\ln \frac{2 l}{w}+0.5+0.178 \frac{w}{l}+0.0146\left(\frac{w}{l}\right)^{2}-\ln \left(\frac{w+t}{w}\right)\right]$

Where 1 and $\mathrm{w}$ are lateral parameters and $\mathrm{t}$ is vertical parameter of ELC structure. 1 is the length of the cell and $w$ is the metal width. $1=3 \mathrm{~mm}, \mathrm{w}=0.25 \mathrm{~mm}$.

The parallel plate capacitance of the slits in the ELC structure can be expressed as:

$$
C=\frac{\varepsilon_{0} A}{d}
$$

Where A is the area of the plate capacitance of the slits and d is the distance between the plates $(\mathrm{d}=\mathrm{g}=0.25 \mathrm{~mm}$, gap width).

\subsection{Patch antenna}

Single patch microstrip antenna is designed to operate at the frequency $14.3 \mathrm{GHZ}$ where the frequency is in the range of negative permittivity and positive permeability. The copper material is used for microstrip antenna and coaxial feed as shown in Figure 4. The dimensions of patch antenna were obtained by applying some basic formula of patch antenna design as given below [8].

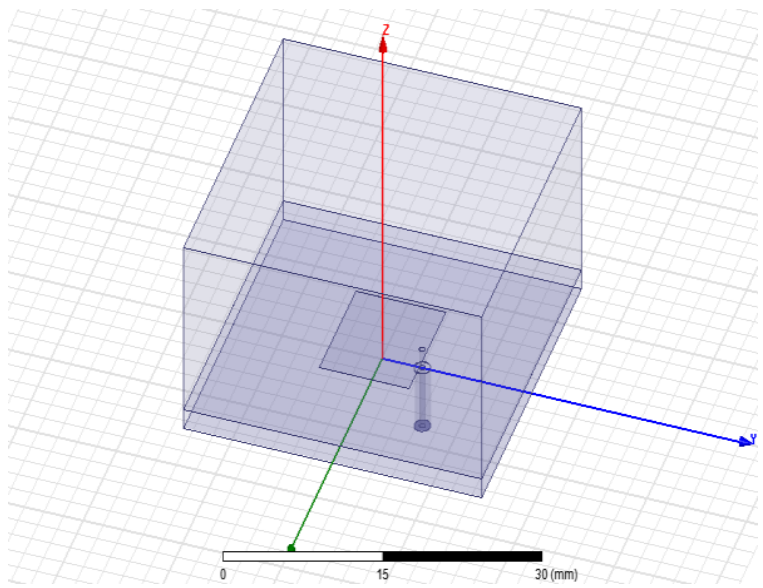

Fig 4: HFSS simulation setup for Microstrip patch antenna

$$
\operatorname{Width}(W)=\frac{C}{2 f_{r} \sqrt{\frac{\varepsilon_{r}+1}{2}}}
$$

Where

$\mathrm{c}=$ free space velocity of light

$\mathrm{f}_{\mathrm{r}}=$ frequency of operation

$\varepsilon_{\mathrm{r}}=$ dielectric constant

1. Effective Dielectric constant:

$$
\varepsilon_{r_{e f f}}=\frac{\varepsilon_{r}+1}{2}+\frac{\varepsilon_{r}-1}{2}\left[1+12 \frac{h}{w}\right]^{-\frac{1}{2}}
$$

Where $\mathrm{h}=$ height of dielectric substrate

$$
\mathrm{w}=\text { width of patch }
$$

2. Effective Length

$$
L_{e f f}=\frac{c}{2 f_{r} \sqrt{\varepsilon_{r_{e f f}}}}
$$

3. Patch Length Extension

$$
\Delta L=0.412 h \frac{\left(\varepsilon_{r_{e f f}}+0.33\right)}{\left(\varepsilon_{r_{\text {eff }}}-0.258\right)} \frac{\left(\frac{w}{h}+0.264\right)}{\left(\frac{w}{h}+0.8\right)} \ldots \ldots \ldots
$$

4. Actual length of patch

$$
\begin{array}{r}
L=L_{\text {eff }}-2 \Delta L . \\
\text { Table } 1
\end{array}
$$

\begin{tabular}{|c|c|}
\hline Properties & Dimensions(mm) \\
\hline Patch Width, $\mathrm{W}$ & $\mathbf{9}$ \\
\hline Patch Length, $\mathrm{L}$ & $\mathbf{1 1}$ \\
\hline Substrate Width, $\mathrm{W}_{\mathrm{g}}$ & $\mathbf{3 0}$ \\
\hline Substrate Length, $\mathrm{L}_{\mathrm{g}}$ & $\mathbf{3 0}$ \\
\hline
\end{tabular}




\subsection{Metamaterial cover}

The unit cells of metamaterial structure are combined to form 3D array shown in Figure 5. Now ENG metamaterial cover is placed in front of microstrip antenna with the optimized distance of $10.52 \mathrm{~mm}$ which is approximately equal to half wavelength. The metamaterial cover incorporated with patch antenna shown in Figure 6.

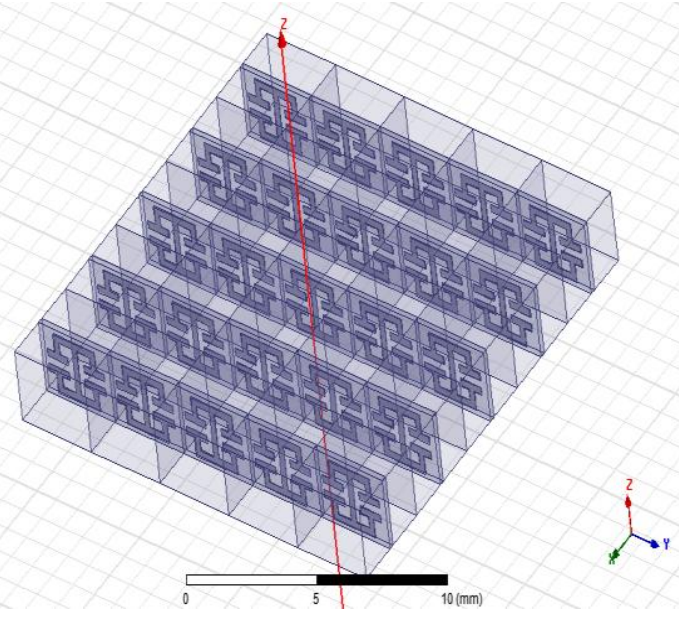

Fig 5: 3 Dimensional array topology

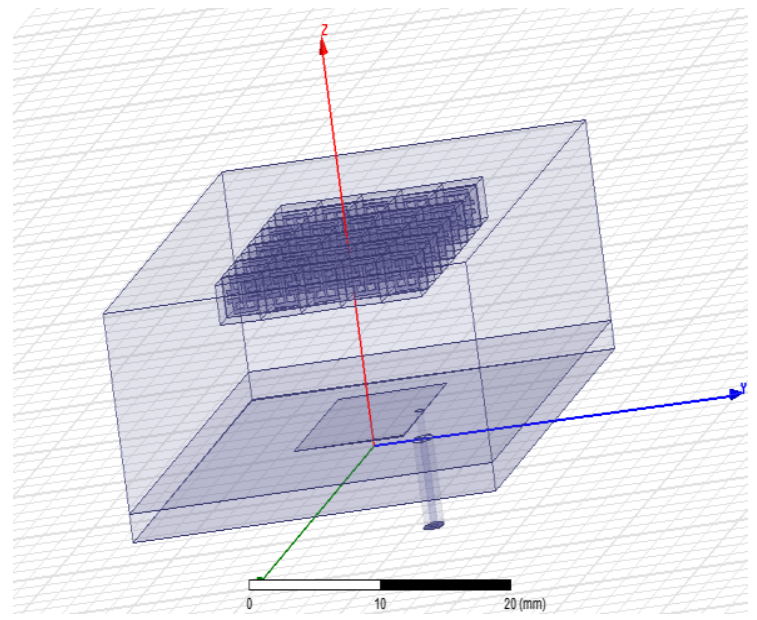

Fig 6: Microstrip patch antenna incorporated with MTM cover

\section{RESULTS}

In the present section we report the simulation result for the ELC unit cell, patch antenna without metamaterial and patch antenna with metamaterial. The resonance frequency of the unit cell using the geometrical and physical parameters specified earlier is estimated to be $\mathrm{f}=14.46 \mathrm{GHZ}$ from the equivalent circuit model approach. By the HFSS simulation the resonance frequency obtained is $14.3 \mathrm{GHZ}$ by less than $5 \%$ error. To show the physical properties of designed structure, the effective material parameter can be extracted from the $\mathrm{S}$ parameters as [9].

$$
z=\frac{\sqrt{\left(1+S_{11}\right)^{2}-\left(S_{21}\right)^{2}}}{\sqrt{\left(1-S_{11}\right)^{2}-\left(S_{21}\right)^{2}}}
$$

$$
n=\frac{1}{k d} \cos ^{-1}\left(\frac{1}{2 S_{21}}\left(1-S_{11}^{2}+S_{21}^{2}\right)\right)
$$

Where $\mathrm{z}$ and $\mathrm{n}$ indicates the wave impedance and refractive index respectively then, the electrical permittivity and magnetic permeability can be computed from the equation of $\varepsilon=\mathrm{n} / \mathrm{z}$ and $\mu=\mathrm{n} * \mathrm{z}$.

In the Figure 7 to Figure 10 the simulation results shows that the structure has the metamaterial property at the resonance frequency.

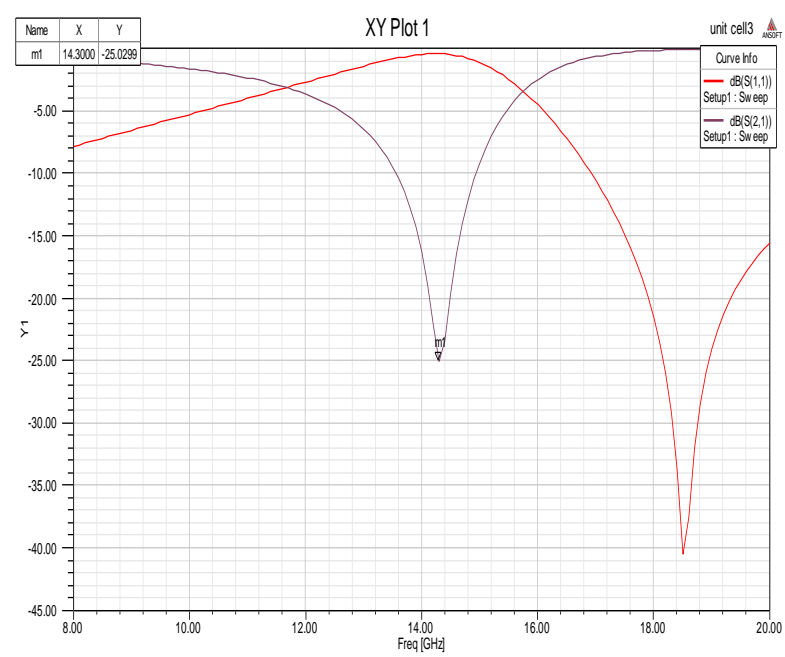

Fig 7: S parameters for the unit cell

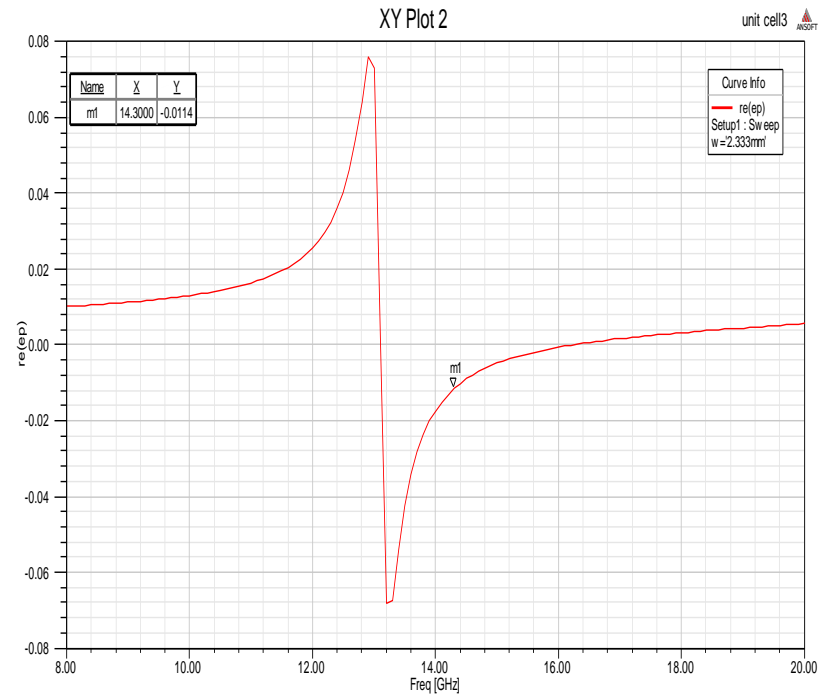

Fig 8: Electric permittivity 


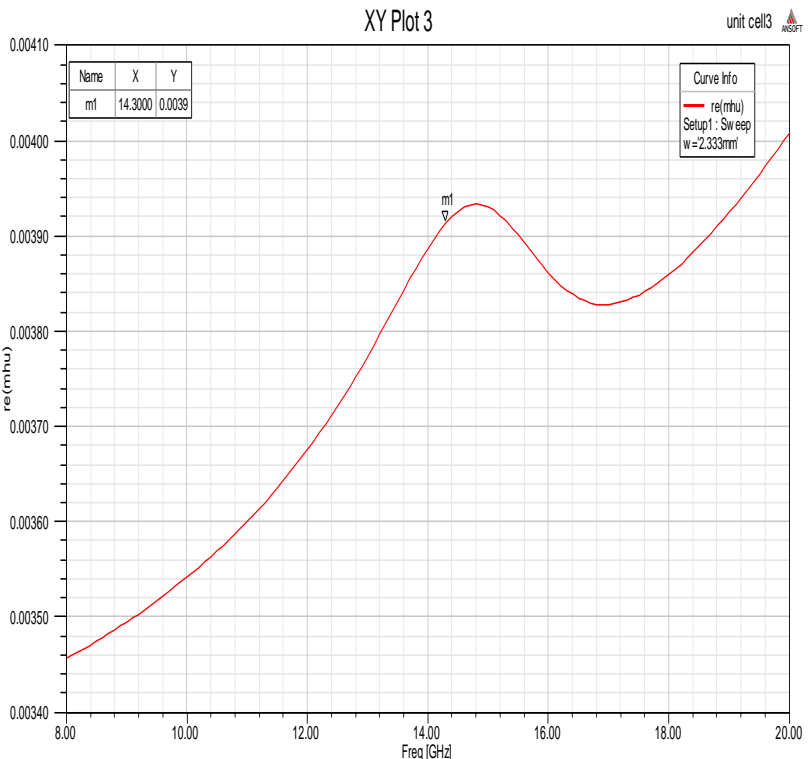

Fig 9: Magnetic permeability

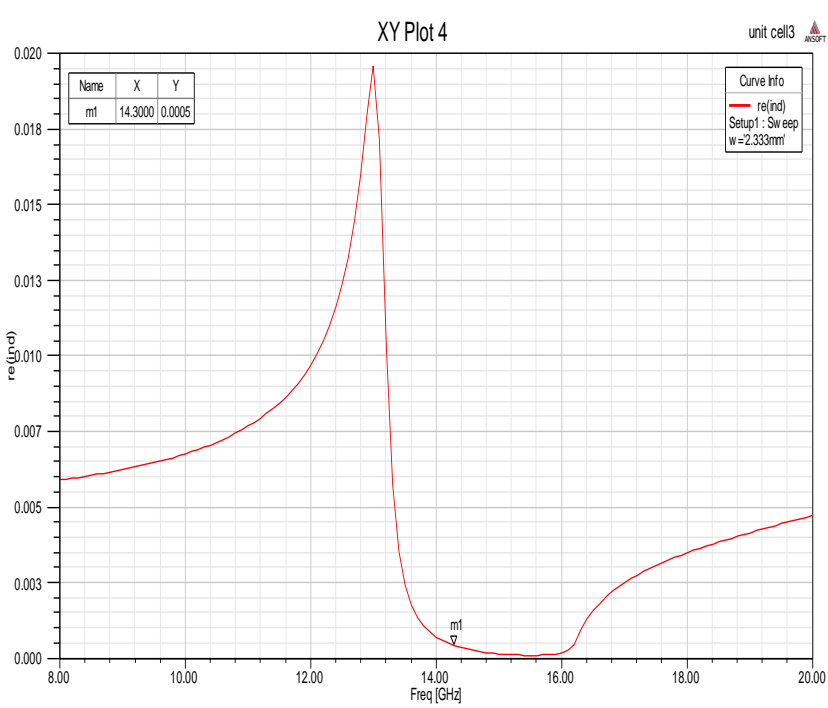

Fig 10: Real part of refrective index

\subsection{Return Loss}

Figure 11 and Figure 12 shows the simulated returned loss of the microstrip antenna without MTM cover and with MTM cover respectively. The return loss shows a good agreement between microstrip antenna with and without ENG metamaterial where both resonance at $14.3 \mathrm{GHZ}$.

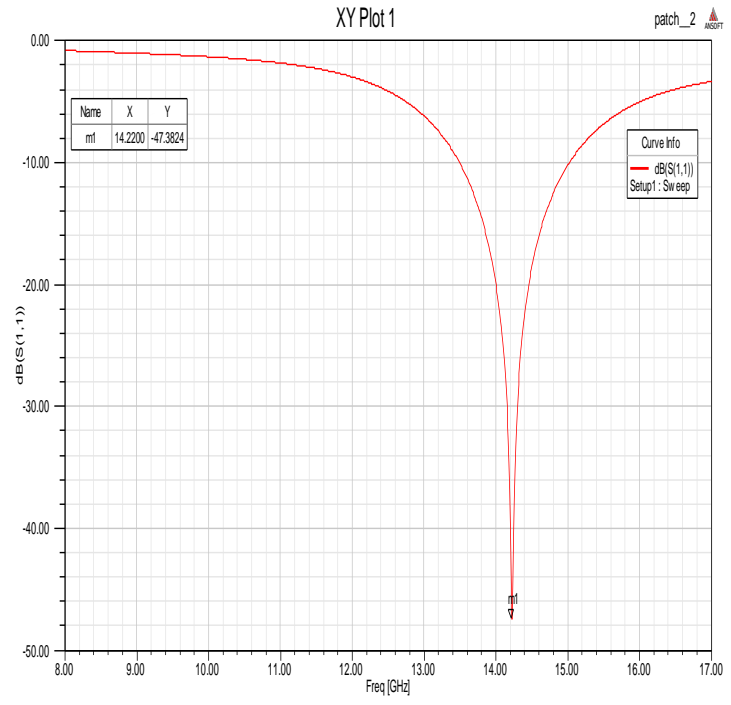

Fig 11: S parameter of microstrip path antenna

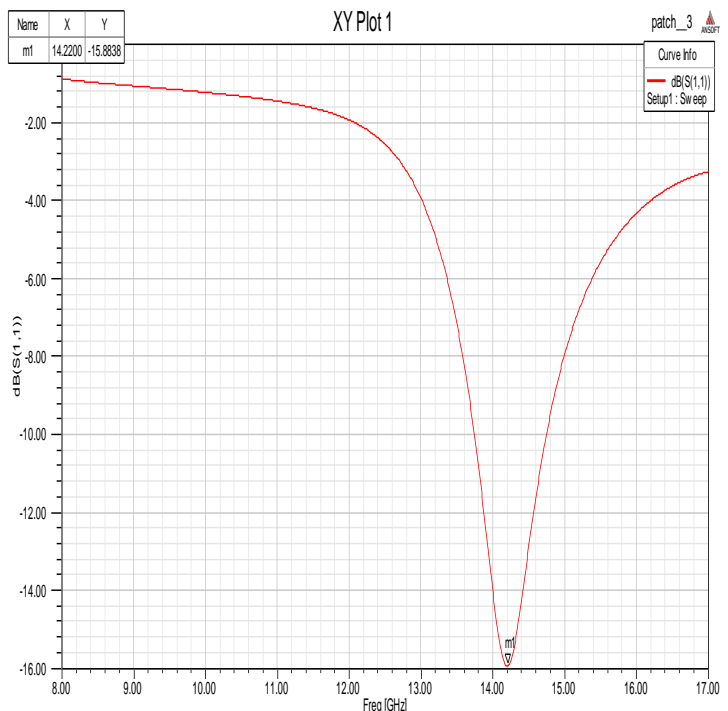

Fig 12: S parameter of MSA incorporated with MTM cover

\subsection{Radiation Pattern}

Figure 13 and Figure 14 shows the radiation pattern of microstrip antenna without metamaterial cover and with MTM cover. The radiation pattern of microstrip antenna without and with metamaterial structure has different gain. In comparisons between gain/directivity of both radiation pattern shows an increasing of gain by attaching the metamaterial structure in front of the microstrip antenna. 


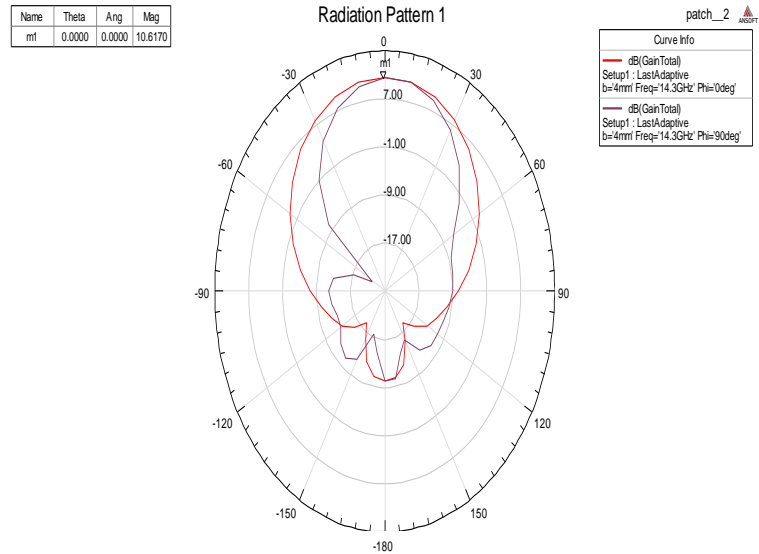

Fig 13: Radiation pattern of MSA without MTM cover

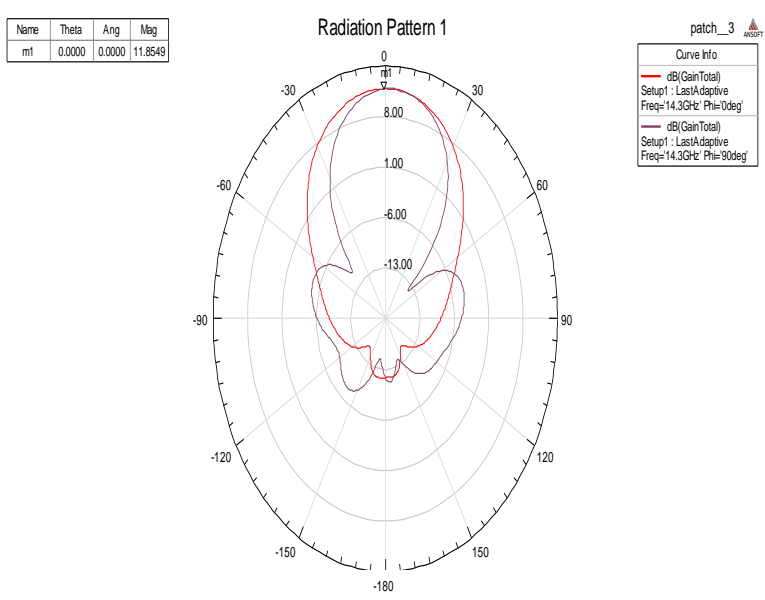

Fig 14: Radiation pattern of MSA incorporated with MTM cover

\subsection{Directive Gain}

A high radiation directivity of an antenna indicates that the antenna has high power intensity. Figure 15 and Figure 16 shows the directive gains of the antenna with or without metamaterial cover. The maximum radiation directivity of convention antenna is $10.48 \mathrm{~dB}$ while a maximum radiation directivity of $11.94 \mathrm{~dB}$ is obtained for antenna with metamaterial cover.

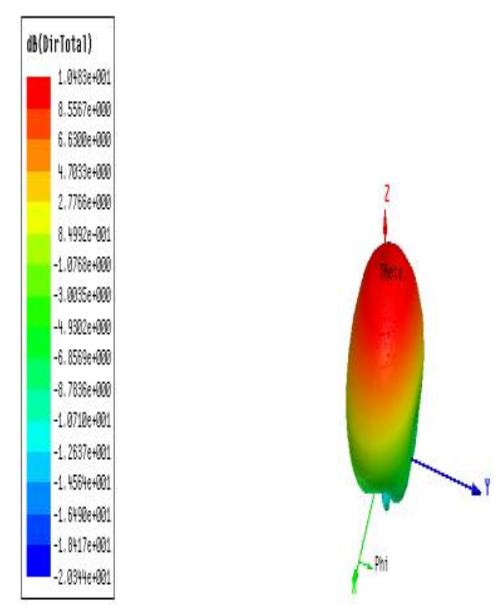

Fig 15: Directivity in 3D pattern of MSA without MTM cover
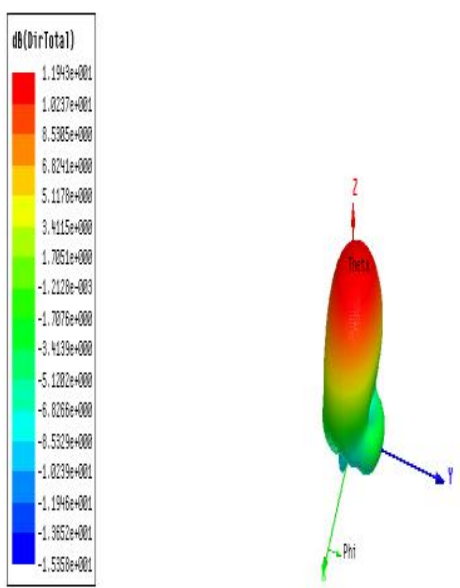

Fig 16: Directivity in 3D pattern of MSA incorporated with MTM cover

\section{CONCLUSION}

From the observation of radiation pattern of microstrip antenna integrated with MTM cover has an improved gain compared to the gain of the microstrip antenna without MTM cover. An improvement of the gain from $10.48 \mathrm{db}$ to $11.94 \mathrm{db}$ in simulation was obtained when MTM cover is placed in front of the microstrip antenna. However, despite increasing of gain, a bigger side and back lobe was also been introduced. If the side and back lobe can be reduce, the Gain of the microstrip antenna with MTM cover can be further improved.

\section{REFERENCES}

[1] Hao Y, Mitra R (2009), "FDTD Modeling of metamaterials", Artech house Norwood, vol.103: 110114.

[2] Jun CT, Smith DR, Liu R (2010), "Metamaterials: Theory, Design, and Applications", Springer New York, pp.3-10.

[3] Richard W. ZIOLKOWSKI (2006), "MetamaterialBased Antennas: Research and Developments," IEICE Trans. Electron.,Vol.E89-C, NO.9, pp. 1267-1275.

[4] Erentok A, Ziolkowski RW (2008), "metamaterial inspired efficient electrically small antennas", IEEE trans. Ant. Prop., vol.56(3): 691-707.

[5] Schuring D, Mock JJ, Smith DR (2006), "Electric-fieldcoupled resonators for negative permittivity metamaterials," journal of applied physics letters, vol.88, 041109.

[6] Ziolkowski RW, Jin P, Nielsen JA, Tanieliam MH, Hollowway CL (2009), "Experiment verification of Z antennas at UHF frequencies", IEEE Antenna wireless Propag. Let. 8: 1329-1333.

[7] Chen H, L. Ran, J. Huang fu, T.M. Grzegorczyk, J. Kong (2006), "equivalent circuit model for left- handed metamaterials", journal of applied physics, vol.100, 024915 .

[8] Z. Awang, Microwave Engineering for Wireless Communications: Prentice Hall, 2006.

[9] Chen,X., T.M. Grzegorczyk, B. -I. Wu, J. Pacheco, and J.A. Kong, " Robust method to retrieve the constitutive effective parameters of metamaterials," physical review E, Vol.70, 016608.1-016608.7, 2004. 